\title{
A CONCEPT FOR PROCESS-ORIENTED INTERDISCIPLINARY TOLERANCE MANAGEMENT CONSIDERING PRODUCTION-SPECIFIC DEVIATIONS
}

\author{
Heling, Bjoern; Oberleiter, Thomas; Rohrmoser, Andreas; Kiener, Christoph; Schleich, \\ Benjamin; Hagenah, Hinnerk; Merklein, Marion; Willner, Kai; Wartzack, Sandro
}

Friedrich-Alexander-Universität Erlangen-Nürnberg

\begin{abstract}
To meet rising customer requirements, increasingly complex products have to be virtually validated. To achieve this within the framework of virtual product development, a wide range of aspects has to be taken into account. In this context, tolerance analysis has established itself as a proven tool to evaluate the consequences of geometric part deviations on geometric product characteristics. Existing approaches, however, do not sufficiently take into account production-specific deviations, leading to time-consuming iterations during the product development process. Therefore, the focus of this contribution is on process-oriented interdisciplinary tolerance management that allows the integration of manufacturing simulations into the tolerance analysis. In contrast to the conventional approach, this novel methodology allows to avoid unnecessary iterations in the context of product development and validation. Following the presentation of the novel procedure, the application on a case study of an Xray shutter is carried out, whereby surrogate models are integrated in order to reduce the computing time.
\end{abstract}

Keywords: Robust design, Tolerance representation and management, Design for X (DfX)

\section{Contact:}

Heling, Bjoern

Friedrich-Alexander-University Erlangen-Nürnberg

Engineering Design

Germany

heling@mfk.fau.de

Cite this article: Heling, B., Oberleiter, T., Rohrmoser, A., Kiener, C., Schleich, B., Hagenah, H., Merklein, M., 


\section{INTRODUCTION}

Today, products have to meet diverse and frequently conflicting requirements. In medical technology, for example, highly complex products that have to meet different international legal requirements are developed. These products have to work at different locations worldwide and have to be brought to product maturity in ever shorter periods of time (Mohedas et al., 2015). A special challenge in this context is the tolerance allocation of components, because component deviations also influence not only the geometry but also the properties of a product. Tolerance management has been established to ensure that all the unique products produced meet the required set properties from a geometric and functional point of view (Schleich and Wartzack, 2014b).

When awarding component tolerances, the tolerance engineer is usually in the area of tension between production and quality assurance. On the one hand, production focuses on the widest possible tolerances, since tight tolerances drive up both production and inspection costs for individual components immensely. On the other hand, quality assurance strives for the tightest possible deviations, as this ensures both subjectively perceived quality and technical product functions (Heling et al., 2016). In order to defuse this conflict of objectives, different areas have been researched in the area of tolerance management in recent decades.

Since the costs are not only defined by the choice of tolerances, but in particular also by the choice of the manufacturing process, this article presents a procedure for taking into account production-specific deviations in the tolerance analysis. A generally applicable transferable procedure is presented and then applied to a case study. This approach does not only deal with the general integration of production-specific knowledge. Rather, a reduction of the computing times by the use of surrogate models is proposed and applied exemplarily. In order to be able to classify the procedure in the research landscape, an overview of relevant research fields in tolerance management is given. In particular, approaches will be discussed that take into account production-specific characteristics.

\section{TOLERANCE MANAGEMENT PROCESS}

The first step the tolerance management process is the description and quantification of the quality feature by Functional Key Characteristics (FKCs) (Thornton, 1999). As shown in Figure 1, the individual part tolerances are then defined, with a preliminary tolerance specification as the starting point. Based on this specification, tolerance analyses are carried out and, depending on whether the desired quality objectives are achieved, tolerance adjustments are performed. If all requirements are met, a final evaluation and documentation of the process takes place (Stuppy, 2011).

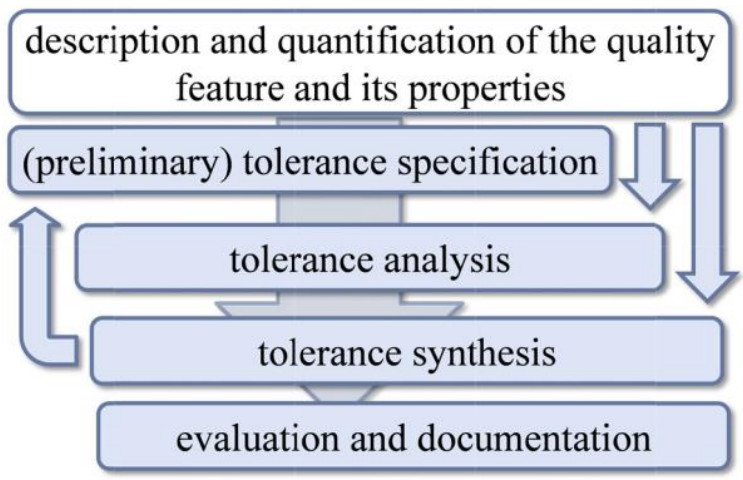

Figure 1. Tolerance Management process (according to (Stuppy, 2011))

In addition to this generic description, other approaches focus on different aspects of the tolerance management process. For example, Mathieu and Marguet (2001) present a method, grounding on the idea that geometric deviations occur along the assembly sequence. Starting from a comparison of different variants, the selection of the best possible assembly sequence is aimed at. Roy et al. (2001) introduce a synthesis process based on the assignment of requirements to geometry elements. In (Dantan et al., 2003) another procedure for the tolerance management process is presented, whereby the focus lies on the concept phase. There are additional approaches that focus on tolerance allocation in early design stages (Goetz et al., 2018). 
Besides the work related to the consideration of processes, other researchers are involved in the improvement of tolerance analysis tools. The main focus here is on the consideration of different possibilities to map tolerances. Examples are extensions of the vectorial tolerance interpretation (Geis et al., 2015), the deviation domain approach (Giordano et al., 2007), the T-Maps $®$ (Ameta et al., 2018) and the concept of Skin Model Shapes (Schleich and Wartzack, 2014a). The improvement of these methods has the consequence that the possibilities of mapping geometric deviations are getting more precise. At the same time, the significance of the input data continues to increase, as the results on tolerance analyses are based on these. Therefore, some research approaches differ in the tolerance analysis by taking into account manufacturing-specific aspects. These approaches will be discussed in the following.

Different approaches focus on the consideration of production-specific aspects in the tolerance analysis. Stockinger and Meerkamm (2009) present an approach that combines sheet metal forming with tolerance analysis. In addition to production influences, the compliance of the individual sheet metal parts are taken into account in the tolerance analysis. Wärmefjord et al. (2010) examine the importance of the welding sequence in spot welding. For this purpose, the tolerance simulation is combined with a welding simulation so that both the sequence of component deviations and process influences are mapped. Since the theoretical number of spot welding combination is extremely high, in the contribution different optimization procedures are used to find the best sequence.

Schleich and Wartzack (2015) present a methodological framework, which focuses on the integration of deviation information from different product development phases. In addition to the general approach, the feasibility is demonstrated on a case study, whereby the results of an injection molding simulation are integrated into the tolerance analysis model.

\section{CONCEPT OF INTERDISCIPLINARY PROCESS-ORIENTED TOLERANCE MANAGEMENT}

Since tolerance management should run as efficiently as possible on the basis of well-founded information due to its many interfaces, the question arises as to how technical production aspects can be taken into account in tolerance management? In order to answer this question, a new procedure model, which takes into account the integration of production influences on component deviations, is described below. Subsequently, the new procedure model is exemplary applied and the advantages as well as the disadvantages of this new approach are discussed.

Figure 2 therefore shows the schematic process of the interdisciplinary process-oriented tolerance management. The starting point is the conventional procedure, in which tolerance values $T_{i}$ are determined based on expert estimates, standards or special tables. Additional reconciliations and insufficient consideration of production-specific geometric deviations are, however, disadvantages associated with this procedure.

In contrast to this conventional approach, interdisciplinary process-oriented tolerance management predicts the expected deviations based on manufacturing process simulations or real production data. Thus, specific deviations of a part manufactured with a certain process can be taken into account from the beginning.

Since very large amounts of data that go along with high computing times are available for simulations as well as for produced pre-series components, surrogate models $y\left(x_{i}\right)$ can optionally be integrated. These surrogate models, which enable a significant reduction in computing time, can then be integrated into the tolerance analysis. In the following, the individual steps of the interdisciplinary process-oriented tolerance management are described in detail. 


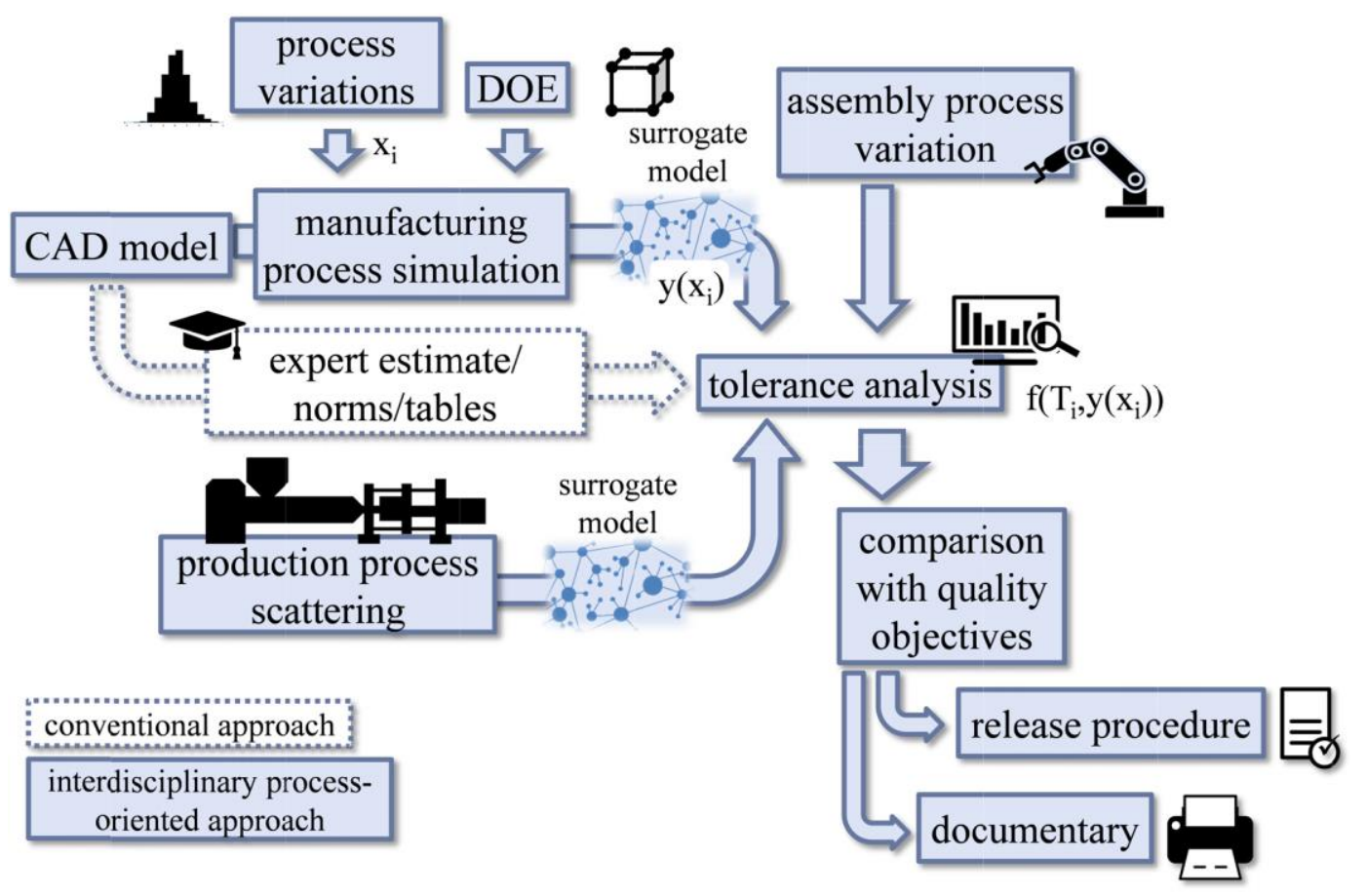

Figure 2. Interdisciplinary process-oriented tolerance management

\subsection{Manufacturing process simulations}

By integrating the manufacturing process simulations, precise, objective tolerance estimations, independent of predecessors can be provided and integrated into the tolerance analysis. However, this presents various challenges that need to be mastered. For example, manufacturing simulations and the corresponding manufacturing processes must both be verified and validated with regard to their quality of results. Consequently, it must not only be clarified to what extent an underlying mathematical model accurately represents the solution, but also to what extent reality is adequately represented by the model.

After the creation of the manufacturing simulation, the results have to be evaluated so that statements about the component deviations can be made. For this purpose, suitable measurement algorithms have to be implemented so that the deviations can be determined in accordance with the GPS standards (ISO 8015, 2011). In this context, it has been shown that the stl-format is particularly well suited as interface between different simulation environments.

The case study in this article deals with cold forged gearwheels. Therefore, a brief description of the manufacturing process will be given. Conventionally the manufacturing of steel gears and geared components is done by machining processes as they enable high geometrical accuracy and flexibility (Klocke et al., 2008). However, the productivity of these machine-cutting processes is limited due to the process-principle and the significant material loss during tooth shaping (Bausch, 2009). In this context, cold forging provides the capability to produce geared components in a highly productive process with excellent material utilization. Disadvantages are the limited geometric accuracy compared to machining processes and the high tooling costs (Lange, 2011).

\subsection{Generation of surrogate models}

In order to represent manufacturing processes with uncertainties of known or even unknown influences and thus simulate a realistic representation, a large number of model evaluations is required. In doing so, uncertain parameters are defined and their range of variation is integrated into the simulation.

With the introduction of variations, the production process simulation must be run through many times. Depending on the complexity of the process simulation, this can result in expensive calculation times that are no longer manageable. 
In such cases, there are several possibilities to reduce the calculation time. Beside model reductions e.g. POD (prober orthogonal decomposition) (Chinesta et al., 2011) there is the possibility to use mathematical replacement models, so-called surrogate models.

There are different approaches like polynomial models, radial basis function models, support vector regression or Kriging (Forrester et al., 2008). In this contribution, a Kriging model is used to represent the manufacturing process of forward extrusion.

This method was developed by Krige (Krige 1951) and extended in (Sacks et al., 1989) for mechanical problems called Design and Analysis of Computer Experiments (DACE). The surrogate model based on evaluation points, which are results of the process simulation. For the calculation of new results with the surrogate model the following applies:

$$
\hat{Y}(x)=\sum_{j=1}^{k} \beta_{j} * f_{j}(x)+Z(x)
$$

The $\hat{Y}(x)$ results from the regression model (1st part) and the random process (2nd part). For $\mathrm{Z}$ a mean value of zero and covariance is assumed. For the variance of two input variables the following results:

$$
V(w, x)=\sigma^{2} R(w, x)
$$

where $\sigma^{2}$ is the process variance and $\mathrm{R}$ is the correlation. For more details, see (Sacks et al., 1989). With such a Kriging model, a simulation can be reproduced, as schematically shown in Figure 3 for the one-dimensional case.

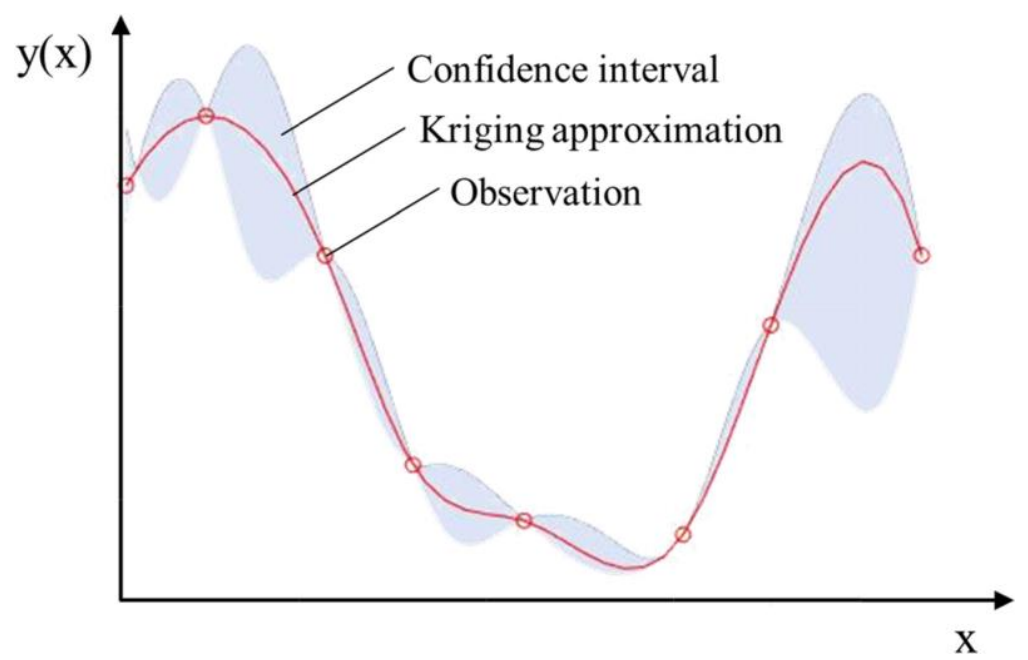

Figure 3. Kriging approximation and the corresponding confidence interval

It becomes clear that the confidence interval is varying in size in different places and corresponds to zero at the observations. This is due to the fact that the surrogate model was adapted to them.

\subsection{Tolerance analysis}

After the generation of the surrogate model, a tolerance analysis model can be built, whereas different forms to represent the geometric deviations can be used. For example, the analysis can be done with vector models, Skin Model Shapes or with the help of commercial feature-based analysis programs, such as 3DCS or Vis VSA (Schleich and Wartzack, 2016). To integrate the surrogate models in the analysis, two fundamentally different approaches can be used. On the one hand, the input variables can be sampled so that the uncertainties are taken into account, during the use of the surrogate model. Thus, the surrogate model would provide a probability distribution of the initial size instead of one single value. This approach should be used if the surrogate model determines a discrete geometry representation, such as a Skin Model. In this case, the surrogate model would be used to create a specific variant of the Skin Model - a so-called Skin Model Shape.

On the other hand, non-varying input variables can be used and sampling is then performed on the basis of the surrogate model's output. This approach, which is shown in Figure 4, enables the 
generation of $n$ individual parts, while the surrogate model has only one scalar output variable. This procedure is useful if the surrogate model describes a scalar size. This can be the case, when geometry deviations are measured on the basis of tests or simulations.
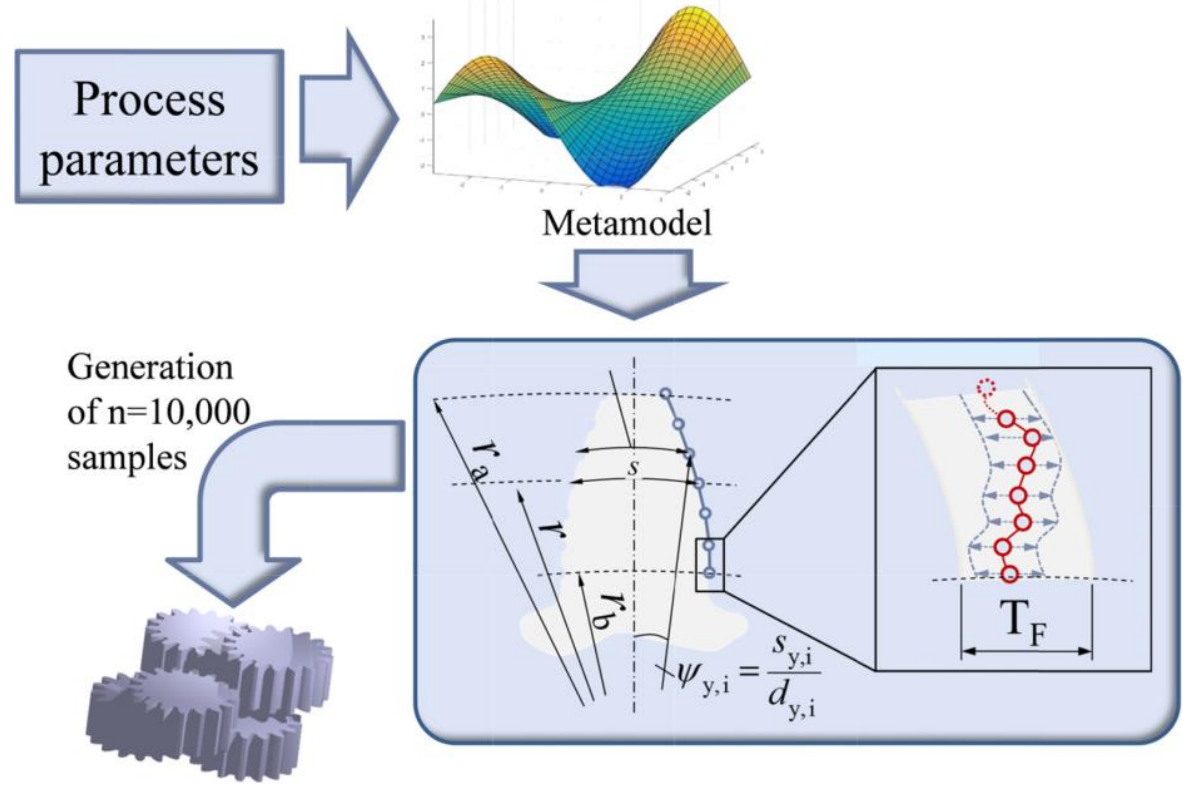

Figure 4. Procedure for generating the pinion

Apart from the two possibilities described above, a combination of both approaches is also conceivable. The result would then be, for example, a Skin Model Shape, which was generated on the basis of scattering input variables. However, this Skin Model Shape is not immediately used for the tolerance simulation. Rather, a second sampling of geometry deviations is generated using this Skin Model Shapes. This approach does justice to the fact that although simulations predict geometrical deviations deterministically, these predictions show a certain uncertainty.

\section{EXEMPLARY APPLICATION OF PROCESS-ORIENTED TOLERANCE MANAGEMENT}

In the following, the steps described above for process-oriented tolerance management are carried out for a specific case study. This involves integrating a surrogate model that maps the massive forming of a gear into a tolerance analysis. The kinematics of the X-ray shutter shown in Figure 5 is used as a demonstrator.

With this kinematic, the X-ray shutter moves on a curve defined by the two articulated levers. The left lever is driven by the connecting lever and a gear. The gear in turn is driven by a motor via a pinion.

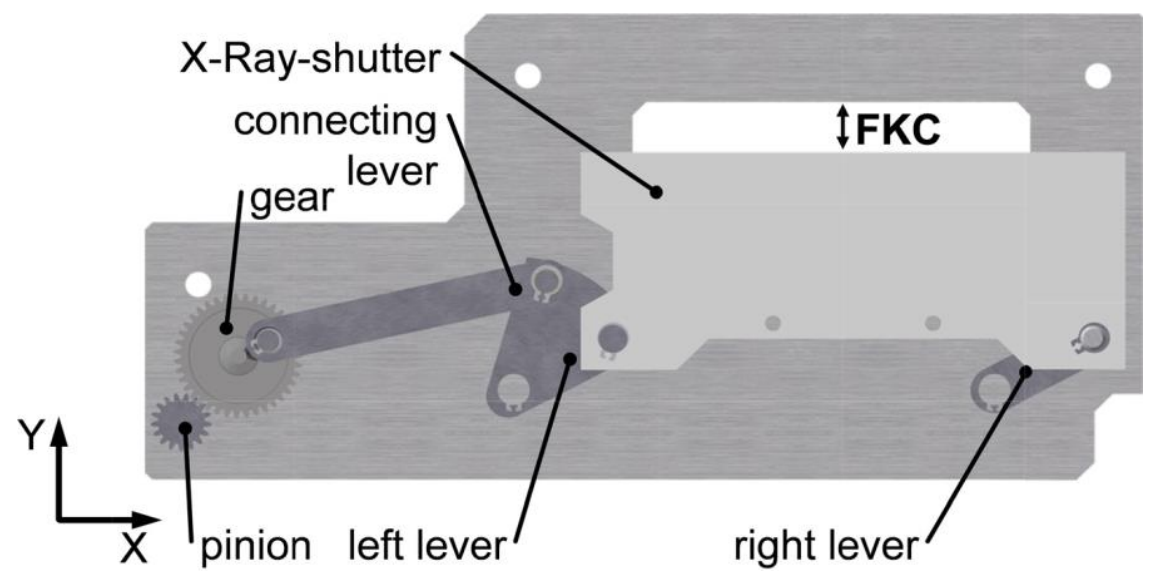

Figure 5. Kinematic of a X-Ray-Shutter (according to Heling et al., 2017) 
The geometric deviations of the pinion are generated on the basis of a surrogate model, which in turn is based on a manufacturing simulation.

\subsection{Manufacturing simulation of extrusion of steel gears}

As described above, the case study uses pinions produced by cold forging. Within this research project the influence of varying process parameters on the resulting geometrical accuracy of a cold forged pinion is investigated. Therefore, the cold forging process was mapped by a FE forming simulation in order to determine the gear geometry and the resulting profile deviation. The required input variables regarding the material behaviour and friction conditions have been determined in laboratory tests (Lorenz et al., 2018). Furthermore, the prognosis quality of the numerical process model was validated by experimental investigations. In this context the associated research group is conducting further detailed studies of the cold forging process, which are described in a separate contribution to this conference. The methodology within the investigation is shown in Figure 6.

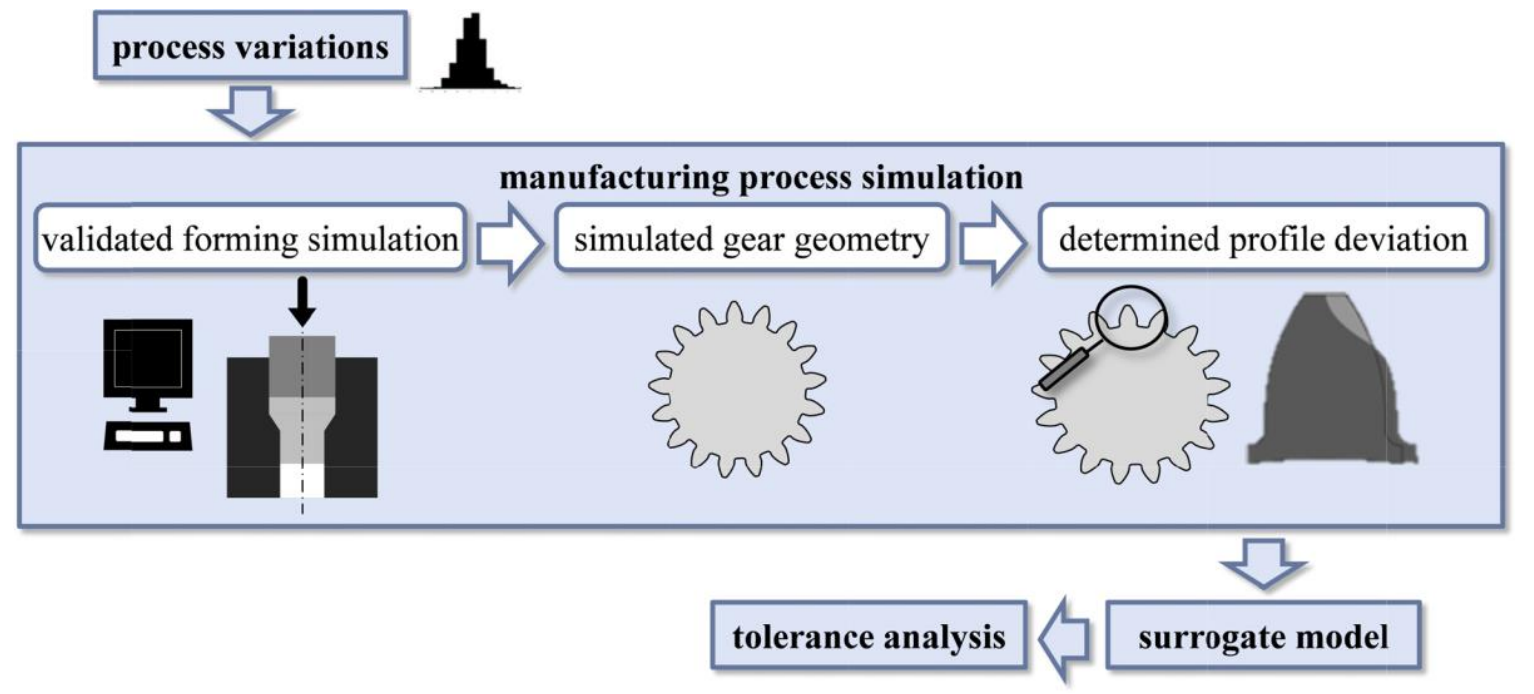

Figure 6. Determination of process related component deviations with the help of a manufacturing process simulation

Important influencing factors are the tribological conditions during forming and the geometry of the workpiece. On that account, the friction factor and the diameter of the workpiece have been varied in the process simulation. The parameter space was chosen to represent process-related fluctuations. On that basis, the resulting gear geometry was determined using the validated simulation model. In the next step, the profile deviation of the simulated tooth geometry were calculated in accordance to ISO 1328 (ISO 1328, 2013) and used as input variable for the surrogate models.

To create the surrogate model, this procedure is carried out for different process constellations. The input variables are varied during the simulation and the resulting profile deviations of the gears are determined.

\subsection{Generation of surrogate models}

The different constellations result from a sampling plan, which systematically records the profile deviation of the pinions. In the present case, a full factorial design is used. For these sample data yields $\hat{y}(x)=y(x)$, as can exemplary be seen in Figure 3. That means, the result of the surrogate model corresponds to the result of the manufacturing simulation. Evaluations for other input parameters $\mathrm{x}$ are then performed using the equation 1 . The regression model corresponds to a firstorder polynomial and the correlation model to an exponential relationship. Thus, the surrogate model maps the expected profile deviation of the pinion.

\subsection{Tolerance analysis}

For the tolerance analysis, the Hybrid Tolerance Representation (HTR) with $n=10,000$ samples is used to analyse the effects of geometric deviations. This analysis model is a combination of vectorial 
and discrete geometry representation and has the advantage that on the one hand higher joint pairs can be mapped with sufficient accuracy and on the other hand time-saving methods such as vector tolerance analysis can be used (Heling et al., 2017).

Since the surrogate model provides the profile deviation as a scalar output variable, sampling is carried out afterwards, so that $\mathrm{n}$ individual gears are generated. As input variables for the surrogate model a coefficient of friction of 0.15 and a diameter deviation of the workpiece of $-20 \mu \mathrm{m}$ is assumed. Besides these deviations from the ideal manufacturing process of the pinion, the case study contains a dimensional $(T 1=0.5 \mathrm{~mm})$ and a profile tolerance $(T 2=1 \mathrm{~mm})$ as shown in Figure 7 . To ensure reproducibility all other dimensions are assumed to be ideal.

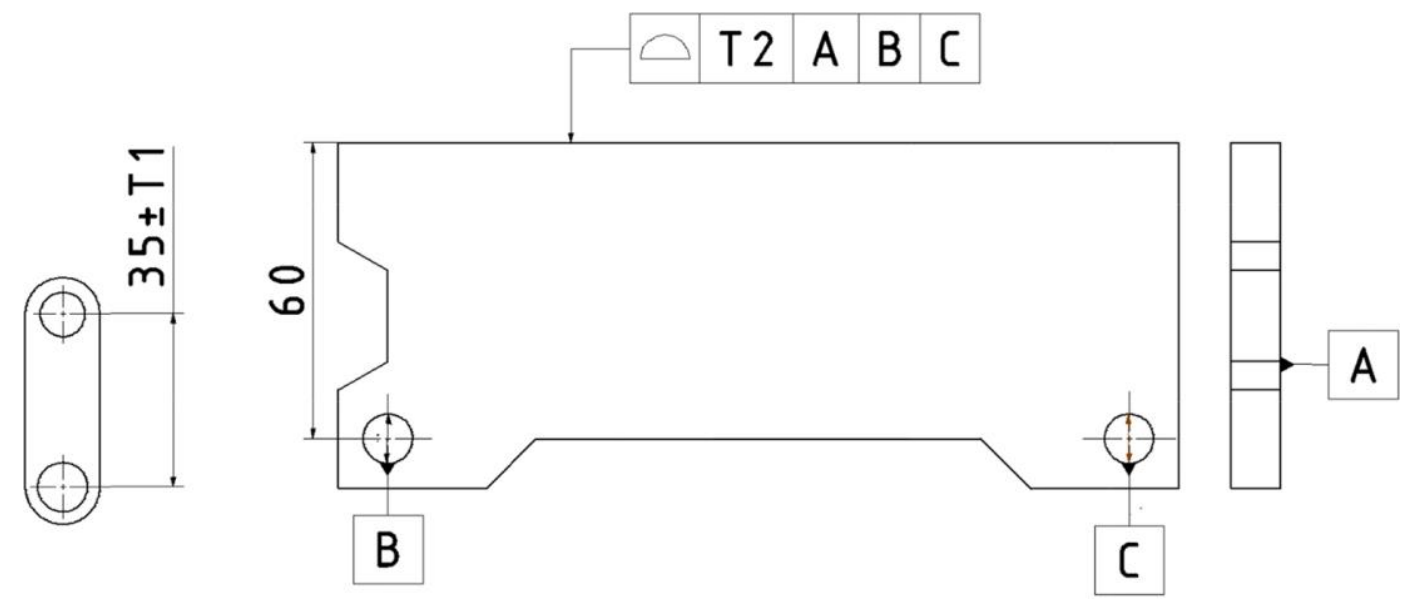

Figure 7. Tolerance specification of X-Ray-Shutter

Consequently the tolerance analysis depends on part Tolerances $T_{i}$ and the integrated surrogate model $y\left(x_{i}\right)$, which indirectly represents the influences of the manufacturing simulation:

$$
f\left(T_{i}, y\left(x_{i}\right)\right) .
$$

\subsection{Results of Tolerance Analysis and their discussion}

The results of the tolerance analysis are shown in Figure 8, where the way in Y-direction (see Figure 5) is plotted in the direction above the rotation angle of the pinion. Each virtual assembly generates a curve that describes the motion behaviour of the X-Ray shutter.

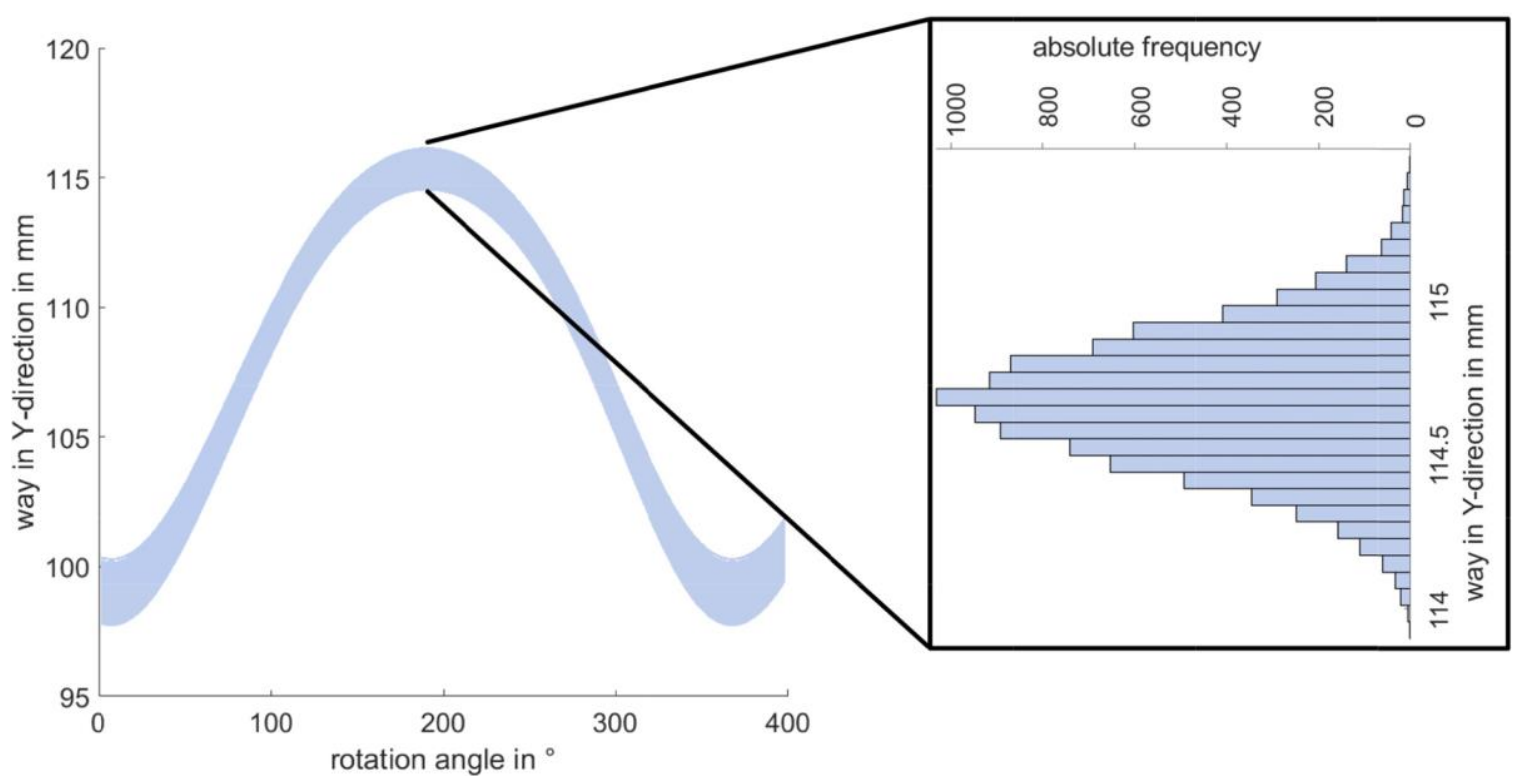

Figure 8. Y-Position of X-Ray shutter and their absolute frequency 
For the $n=10,000$ virtual assemblies, this results in 10,000 superimposed curves. This superposition becomes clear when looking at a special angle. Therefore the frequency distribution of the FKC is shown for an angle of $180^{\circ}$.

A closer look reveals that the scattering is not the same for every angular position. Rather, it fluctuates between $0.22 \mathrm{~mm}$ and $0.33 \mathrm{~mm}$, depending on the angle of rotation. When considering the reasons for the dispersion of the FKC, this can be attributed on the one hand to the deviations of the pinion and on the other hand to the deviations of the right lever and the X-Ray shutter itself.

Although the use of the metamodel for tolerance analysis is time-consuming during initial creation, the successful integration of the metamodel could be demonstrated during tolerance analysis.

On the one hand, the use of surrogate models in tolerance analysis is time-consuming. In a first step, a simulation model must be validated and evaluated at suitable points. Subsequently, sampling takes place in which $\mathrm{n}$ individual Skin Model Shapes are generated, which in turn are integrated into the tolerance analysis. On the other hand, the integration of manufacturing process simulations offers decisive advantages. For example, process-specific characterisitcs can be mapped with this procedure. The procedure also offers the option of selecting the production process windows that are useful from the point of view of tolerance analysis as part of an optimization.

\section{SUMMARY AND OUTLOOK}

In the course of the tolerance management process, it is always necessary to balance between tight costintensive and wide tolerances - which may reduce the quality of a product. Since decisions are made on the basis of simulation results, it is crucial that specific process characteristics are mapped in the best possible way. Therefore, this paper presents an universal, transferable approach that allows this: the interdisciplinary process-oriented tolerance management. With this approach, production-specific deviations can be integrated into the tolerance analysis with little computational effort, using surrogate models. This novel approach was successfully applied in this paper on a case study. In this case study, a validated extrusion process is integrated into the tolerance analysis with the help of a kriging surrogate model.

The successful integration of manufacturing simulation into the tolerance analysis also opens up new potential for further research work. With the integration of measurement uncertainties, for example, further practice-relevant uncertainties could be integrated into the tolerance analysis. The virtual mapping of assembly processes also represents an interesting field of research, since these can add further deviations but can also compensate certain geometric deviations.

\section{REFERENCES}

Ameta, G., Singh, G., Davidson, J.K. and Shah, J.J. (2018), “Tolerance-Maps to Model Composite Positional Tolerancing for Patterns of Features", Journal of Computing and Information Science in Engineering, Vol. 18 No. 3, pp. 31003.

Bausch, T. (2009), Innovative Zahnradfertigung: Verfahren, Maschinen und Werkzeuge zur kostengünstigen Herstellung von Stirnrädern mit hoher Qualität, Kontakt \& Studium, Vol. 175, Expert, Renningen.

Chinesta, F., Ammar, A., Leygue, A. and Keunings, R. (2011), "An overview of the proper generalized decomposition with applications in computational rheology”, Journal of Non-Newtonian Fluid Mechanics, Vol. 166 No. 11, pp. 578-592.

Dantan, J.-Y., Anwer, N. and Mathieu, L. (2003), "Integrated Tolerancing Process for conceptual design", CIRP Annals, Vol. 52 No. 1, pp. 135-138.

Forrester, A.I.J., Sóbester, A. and Keane, A.J. (2008), Engineering design via surrogate modelling: A practical guide, 1st ed., Wiley, Chichester.

Geis, A., Husung, S., Oberänder, A., Weber, C. and Adam, J. (2015), "Use of Vectorial Tolerances for Direct Representation and Analysis in CAD-systems", Procedia CIRP, Vol. 27, pp. 230-240.

Giordano, M., Samper, S. and Petit, J.P. (2007), "Tolerance Analysis and Synthesis by Means of Deviation Domains, Axi-Symmetric Cases", In: J.K. Davidson, (Ed.), Models for Computer Aided Tolerancing in Design and Manufacturing, Springer Netherlands, Dordrecht, pp. 85-94.

Goetz, S., Schleich, B. and Wartzack, S. (2018), "A new approach to first tolerance evaluations in the conceptual design stage based on tolerance graphs", Procedia CIRP, Vol. 75, pp. 167-172.

Heling, B., Aschenbrenner, A., Walter, M. and Wartzack, S. (2016), “On Connected Tolerances in Statistical Tolerance-Cost-Optimization of Assemblies with Interrelated Dimension Chains", Procedia CIRP, Vol. 43, pp. 262-267. 
Heling, B., Hallmann, M. and Wartzack, S. (2017), "Hybrid Tolerance Representation of Systems in Motion", Procedia CIRP, Vol. 60, pp. 50-55.

ISO 1328-1 (2013), Cylindrical gears - ISO system of flank tolerance classification - Part 1: Definitions and allowable values of deviations relevant to flanks of gear teeth No. 1328-1:2013.

ISO 8015 (2011), Geometrical product specifications (GPS) - Fundamentals - Concepts, principles and rules No. ISO 8015.

Klocke, F., Gorgels, C., Kauffmann, P., Herzhoff, S., Schalster, R., Stuckenberg, A. and Vasiliou, V. "Trends in der Zahnradfertigung", In: R. Neugebauer, (Hrsg.), Tagungsband zum 5. Chemnitzer

Produktionstechnischen Kolloquium CPK: Zerspanung in Grenzbereichen, Berichte aus dem IWU, Band 46 2008, pp. 87-113.

Krige, D.G. (1951), "A statistical approach to some basic mine valuation problems on the Witwatersrand", Journal of the Southern African Institute of Mining and Metallurgy, Vol. 52 No. 6, pp. 119-139.

Lange, K. (2011), Handbook of metal forming, Society of Manufacturing Engineers, Dearborn, Mich.

Lorenz, R., Hagenah, H. and Merklein, M. (2018), "Experimental Evaluation of Cold Forging Lubricants Using Double-Cup-Extrusion-Tests", Resource Efficient Material and Forming Technologies, Trans Tech Publications, pp. 65-70.

Mathieu, L. and Marguet, B. (2001), "Integrated Design Method to Improve Producibility based on Product Key Characteristics and Assembly Sequences", CIRP Annals, Vol. 50 No. 1, pp. 85-88.

Mohedas, I., Sabet Sarvestani, A., Daly, S.R. and Sienko, K.H. (2015), Applying design ethnography to product evaluation: A case example of a medical device in a low-resource setting, Vol. 2015.

Roy, U., Pramanik, N., Sudarsan, R., Sriram, R.D. and Lyons, K.W. (2001), "Function-to-form mapping. Model, representation and applications in design synthesis", Computer-Aided Design, Vol. 33 No. 10, pp. 699-719.

Sacks, J., Welch, W.J., Mitchell, T.J. and Wynn, H.P. (1989), "Design and Analysis of Computer Experiments", Statistical Science, Vol. 4 No. 4, pp. 409-423.

Schleich, B. and Wartzack, S. (2015), “A generic approach to sensitivity analysis in geometric variations management", Proceedings of the International Conference on Engineering Design, ICED, pp. 343-352.

Schleich, B. and Wartzack, S. (2014a), "A discrete geometry approach for tolerance analysis of mechanism", Mechanism and Machine Theory, Vol. 77, pp. 148-163.

Schleich, B. and Wartzack, S. (2014b), "How can Computer Aided Tolerancing Support Closed Loop Tolerance Engineering?", Procedia CIRP, Vol. 21, pp. 312-317.

Schleich, B. and Wartzack, S. (2016), “A Quantitative Comparison of Tolerance Analysis Approaches for Rigid Mechanical Assemblies”, Procedia CIRP, Vol. 43, pp. 172-177.

Stockinger, A. and Meerkamm, H. (2009), “Concept for the Integration of Manufacturing Simulations into Tolerance Analysis", 2009.

Stuppy, J. (2011), Methodische und rechnerunterstützte Toleranzanalyse für bewegte technische Systeme, Fortschritt-berichte VDI. Reihe 20, Rechnerunterstütze Verfahren, Nr. 433, VDI, Düsseldorf.

Thornton, A.C. (1999), "A Mathematical Framework for the Key Characteristic Process", Research in Engineering Design, Vol. 11 No. 3, pp. 145-157.

Wärmefjord, K., Söderberg, R. and Lindkvist, L. (November 12-18, 2010), Strategies for Optimization of Spot Welding Sequence With Respect to Geometrical Variation in Sheet Metal Assemblies, Vancouver, British Columbia, Canada.

\section{ACKNOWLEDGMENTS}

The authors would like to thank the German Research Foundation (DFG) for supporting the research project "FOR 2271 process-oriented tolerance management based on virtual computer-aided engineering tools" under grant numbers WA2913/19-1, ME 2043/55-1 and WI1181/8-1. 